\title{
Novel copolymers of vinyl acetate. 4. Halogen ring-substituted ethyl 2-
}

\section{cyano-3-phenyl-2-propenoates}

Benjamin Y. Killam, Rebecca J. Amend, Margaret A. Budz, Ashley B. Collins, Shannon M. Hinz, Amanda C. Johnson, Lesli N. Kibler, Sirena Z. Salem, British A. Scott, Irene Skukan, Erin K. Staunton, Katherine J. Sweeney, Andrew Strang, Rhiannon E. Tennant, Daniel Weber, Ayesha Z. Worsham, and Gregory B. Kharas*

DePaul University, Chemistry and Biochemistry Department, 1110 West Belden Avenue, Chicago, IL 60614-3214

Contact: gkharas@depaul.edu

Novel copolymers of vinyl acetate and halogen ring-substituted ethyl 2-cyano-3-phenyl-2propenoates, $\mathrm{RPhCH}=\mathrm{C}(\mathrm{CN}) \mathrm{CO}_{2} \mathrm{C}_{2} \mathrm{H}_{5}$ (where $\mathrm{R}$ is 2- $\mathrm{Br}, 3-\mathrm{Br}, 4-\mathrm{Br}, 2-\mathrm{Cl}, 3-\mathrm{Cl}, 4-\mathrm{Cl}, 2-\mathrm{F}$, 3-F, 4-F, 2,3-dichloro, 2,4-dichloro, 2,6-dichloro, 3,4-dichloro, 2,4-difluoro, 2-bromo-3,4dimethoxy) were prepared in solution with radical initiation at $70^{\circ} \mathrm{C}$. The propenoates were synthesized by the piperidine catalyzed Knoevenagel condensation of halogen ringsubstituted benzaldehydes and ethyl cyanoacetate, and characterized by CHN analysis, IR, ${ }^{1} \mathrm{H}$ and ${ }^{13} \mathrm{C}-\mathrm{NMR}$. The compositions of the copolymers were calculated from nitrogen analysis and the structures were analyzed by IR, ${ }^{1} \mathrm{H}$ and ${ }^{13} \mathrm{C}-\mathrm{NMR}$. Thermal behavior of the copolymers was studied by DSC (Tg) and TGA. Decomposition of the copolymers in 
nitrogen occurred in two steps, first in the $159-500^{\circ} \mathrm{C}$ range with residue (8.8-15.2 wt\%), which then decomposed in the $500-650^{\circ} \mathrm{C}$ range.

\section{Introduction}

Functionalization of commercial polymers via copolymerization leads to a variety of polymers with interesting properties. Halogen ring-substituted ethyl 2-cyano-3-phenyl-2propenoates (HECP) offer a convenient way to introduce a variety of functional groups in polyvinyl acetate backbone. 2-Bromo HECP is reported in preparation of highly loaded MWCNT-polyamine hybrids and their application in catalysis [1], as well as in synthesis of thiazacridine derivatives as anticancer agents against breast and hematopoietic neoplastic cells [2]. 3-Bromo HECP is involved in synthesis of novel selenophenes from activated acetylenes, ethyl 2-cyano-3-arylacrylate and potassium selenocyanate [3], also in straightforward preparation of highly loaded MWCNT-polyamine hybrids and their application in catalysis [4]. The report on design, synthesis, anti-proliferative evaluation and cell cycle analysis of hybrid 2-quinolones cites 2-chloro HECP [5]. Novel synthesis of 3cyano-2-pyridones derivatives catalyzed by $\mathrm{Au}-\mathrm{Co} / \mathrm{TiO}_{2}$ involves 3-chloro HECP [6]. This HECP also was involved in the study of selenotungstates incorporating organophosphonate ligands and metal ions describing synthesis, characterization, magnetism and catalytic efficiency in the Knoevenagel condensation reaction [7]. 4-Chloro HECP was reported in the study new hybrid organic-inorganic multifunctional materials based on polydopaminelike chemistry [8]. 2-Fluoro HECP was mentioned in study of optimized monofluoromethylsulfonium reagents for fluoromethylene-transfer chemistry [9]. 
Discovery, stereospecific characterization and peripheral modification of 1-(pyrrolidin-1ylmethyl)-2-[(6-chloro-3-oxo-indan)-formyl]-1,2,3,4-tetrahydroisoquinolines as novel selective $\kappa$ opioid receptor agonists involves 3-fluoro HECP [10]. 4-Fluoro HECP was mentioned in study of aerobic oxidative cleavage of $\mathrm{C}=\mathrm{C}$ bond to carbonyl compound [11]. 2,3-Dichloro HECP was reported in synthesis of thiazacridine derivatives as anticancer agents against breast and hematopoietic neoplastic cells [12], whereas 2,4-dichloro HECP was in synthesis of some new dihydropyrimidine derivatives by cyclization of polarized unsaturated systems [13]. Synthesis of multifunctional polymer containing Ni-Pd NPs via thiol-ene reaction for one-pot cascade reactions mentioned 2,6-dichloro HECP [14]. 3,4dichloro HECP was reported in a highly efficient protocol for the regio- and stereo-selective synthesis of spiro pyrrolidine and pyrrolizidine derivatives by multicomponent reaction [15]. 2,4-Difuoro HECP was involved in preparation of indolyl acrylates and related compounds alone and in compositions and methods for modulating hair growth [16]. In continuation of our studies of radical copolymerization of vinyl acetate and arylsubstituted esters 2-cyano-3-phenyl-2-propenoic acid, we have prepared halogen ringsubstituted ethyl 2-cyano-3-phenyl-2-propenoates, $\mathrm{RPhCH}=\mathrm{C}(\mathrm{CN}) \mathrm{CO}_{2} \mathrm{C}_{2} \mathrm{H}_{5}$ (where $\mathrm{R}$ is 2-Br, 3-Br, 4-Br, 2-Cl, 3-Cl, 4-Cl, 2-F, 3-F, 4-F, 2,3-dichloro, 2,4-dichloro, 2,6-dichloro, 3,4-dichloro, 2,4-difluoro, 2-bromo-3,4-dimethoxy) and copolymerized them with vinyl acetate. We reported syntheses of 2-Cl, 3-Cl, 4-Cl, 2-Br, 3-Br, 4-Br, 2-F, 3-F, 4-F, 2-Cl-4F, 2-Cl-6-F, 3-Cl-4-F ring-substituted HECP and copolymerization with styrene [17]. 
There are no reports on synthesis of 2-bromo-3,4-dimethoxy HECP. To the best of our knowledge, there have been no reports on copolymerization of these ethyl 2-cyano-3phenyl-2-propenoates with vinyl acetate [18].

\section{Experimental}

2-Br, 3-Br, 4-Br, 2-Cl, 3-Cl, 4-Cl, 2-F, 3-F, 4-F, 2,3-dichloro, 2,4-dichloro, 2,6-dichloro, 3,4-dichloro, 2,4-difluoro, 2-bromo-3,4-dimethoxy-substituted benzaldehydes, ethyl cyanoacetate, piperidine, vinyl acetate, 1,1'-azobiscyclohexanecarbonitrile, $(\mathrm{ABCN})$, and toluene supplied from Sigma-Aldrich Co., were used as received. Instrumentation is described in [19].

\section{Synthesis of Monomers}

The halogen ring-substituted ethyl 2-cyano-3-phenyl-2-propenoates (HECP) were synthesized by Knoevenagel condensation [20] of a ring-substituted benzaldehyde with ethyl cyanoacetate, catalyzed by base, piperidine. The preparation procedure was essentially the same for all the monomers [19].

$$
\mathrm{RPhCHO}+\mathrm{NCCH}_{2} \mathrm{CO}_{2} \mathrm{C}_{2} \mathrm{H}_{5} \rightarrow \mathrm{RPhCH}=\mathrm{C}(\mathrm{CN}) \mathrm{CO}_{2} \mathrm{C}_{2} \mathrm{H}_{5}
$$

where $\mathrm{R}$ is 2- $\mathrm{Br}$, 3-Br, 4- $\mathrm{Br}$, 2-Cl, 3-Cl, 4-Cl, 2-F, 3-F, 4-F, 2,3-dichloro, 2,4-dichloro, 2,6dichloro, 3,4-dichloro, 2,4-difluoro, 2-bromo-3,4-dimethoxy.

\subsection{Ethyl 2-cyano-3-(2-bromophenyl)-2-propenoate}

Yield 84\%; mp 73.3 $3^{\circ} \mathrm{C},{ }^{1} \mathrm{H}-\mathrm{NMR} \delta 8.1$ (s, 4H, CH=), 7.6-7.4 (m, 4H, Ph), $4.3(\mathrm{t}, 2 \mathrm{H}$,

$\left.\mathrm{OCH}_{2}\right), 1.3\left(\mathrm{t}, 3 \mathrm{H}, \mathrm{OCH}_{2} \mathrm{C}_{3}\right) ;{ }^{13} \mathrm{C}-\mathrm{NMR} \delta 163(\mathrm{C}=\mathrm{O}), 152(\mathrm{HC}=), 138,133,131,130$, $128(\mathrm{Ph}), 116(\mathrm{CN}), 106(\mathrm{C}=), 61\left(\mathrm{OCH}_{2}\right), 14\left(\mathrm{OCH}_{2} \mathrm{CH}_{3}\right) ; \mathrm{IR}\left(\mathrm{cm}^{-1}\right): 3011-2830(\mathrm{~m}, \mathrm{C}-$ 
H), 2223 (m, CN), 1745 (s, C=O), 1595 (C=C), 1253 (s, C-O-CH ( $_{3}, 790-729$ (s, C-H out of plane). Anal. Calcd. for $\mathrm{C}_{12} \mathrm{H}_{10} \mathrm{BrNO}_{2}$ : C, 51.45; H, 3.60; N, 5.00; Found: C, 51.41; H, $3.54 ; \mathrm{N}, 4.90$.

\subsection{Ethyl 2-cyano-3-(3-bromophenyl)-2-propenoate}

Yield $72 \%$; mp $100.5^{\circ} \mathrm{C},{ }^{1} \mathrm{H}-\mathrm{NMR} \delta 8.2(\mathrm{~s}, 1 \mathrm{H}, \mathrm{CH}=), 7.8-7.3(\mathrm{~m}, 4 \mathrm{H}, \mathrm{Ph}), 4.3(\mathrm{t}, 2 \mathrm{H}$, $\left.\mathrm{OCH}_{2}\right), 1.3\left(\mathrm{t}, 3 \mathrm{H}, \mathrm{OCH}_{2} \underline{\mathrm{CH}}_{3}\right) ;{ }^{13} \mathrm{C}-\mathrm{NMR} \delta 163(\mathrm{C}=\mathrm{O}), 154(\mathrm{HC}=), 136,130,133,122$ $(\mathrm{Ph}), 116(\mathrm{CN}), 103(\mathrm{C}=), 61\left(\mathrm{OCH}_{2}\right), 14\left(\mathrm{OCH}_{2} \underline{\mathrm{CH}}_{3}\right)$; IR $\left(\mathrm{cm}^{-1}\right): 3045-2869(\mathrm{~m}, \mathrm{C}-\mathrm{H})$, 2223 (m, CN), 1734 (s, C=O), 1599 (C=C), 1244 (s, C-O-CH 3$), 854$ (s, C-H out of plane). Anal. Calcd. for $\mathrm{C}_{13} \mathrm{H}_{10} \mathrm{BrNO}_{2}$ : C, 52.45; H, 3.60; N, 5.00; Found: C, 51.42; H, $3.53 ; \mathrm{N}, 5.01$.

\subsection{Ethyl 2-cyano-3-(4-bromophenyl)-2-propenoate}

Yield $82 \%$; mp $98.1^{\circ} \mathrm{C},{ }^{1} \mathrm{H}-\mathrm{NMR} \delta 8.2(\mathrm{~s}, 1 \mathrm{H}, \mathrm{CH}=), 7.6,7.5(\mathrm{~d}, 4 \mathrm{H}, \mathrm{Ph}), 4.3(\mathrm{t}, 2 \mathrm{H}$, $\left.\mathrm{OCH}_{2}\right),\left(\mathrm{t}, 3 \mathrm{H}, \mathrm{OCH}_{2} \underline{\mathrm{H}}_{3}\right) ;{ }^{13} \mathrm{C}-\mathrm{NMR} \delta 163(\mathrm{C}=\mathrm{O}), 154(\mathrm{HC}=), 133,132,131(\mathrm{Ph}), 116$ (CN), $133(\mathrm{C}=), 61\left(\mathrm{OCH}_{2}\right), 14\left(\mathrm{OCH}_{2} \underline{\mathrm{CH}_{3}}\right)$; IR $\left(\mathrm{cm}^{-1}\right): 3061-2934(\mathrm{~m}, \mathrm{C}-\mathrm{H}), 2222(\mathrm{~m}$, CN), 1737 (s, C=O), 1243 (s, C-O-C), 844, 759 (s, C-H out of plane). Anal. Calcd. for $\mathrm{C}_{12} \mathrm{H}_{10} \mathrm{BrNO}_{2}$ : C, 51.45; H, 3.60; N, 5.00; Found: C, 51.42; H, 3.35; N, 4.89.

\subsection{Ethyl 2-cyano-3-(2-chlorophenyl)-2-propenoate}

Yield $72 \%$; mp 59.2 ${ }^{\circ} \mathrm{C},{ }^{1} \mathrm{H}-\mathrm{NMR} \delta 8.7(\mathrm{~s}, 1 \mathrm{H}, \mathrm{CH}=), 8.1-7.0(\mathrm{~m}, 4 \mathrm{H}, \mathrm{Ph}), 4.3(\mathrm{t}, 2 \mathrm{H}$, $\left.\mathrm{OCH}_{2}\right), 1.3\left(\mathrm{t}, 3 \mathrm{H}, \mathrm{OCH}_{2} \underline{\mathrm{H}}_{3}\right) ;{ }^{13} \mathrm{C}-\mathrm{NMR} \delta 163(\mathrm{C}=\mathrm{O}), 152(\mathrm{HC}=), 137,132,131,130$, $128(\mathrm{Ph}), 116(\mathrm{CN}), 106(\mathrm{C}=), 61\left(\mathrm{OCH}_{2}\right), 14\left(\mathrm{OCH}_{2} \underline{\mathrm{CH}}_{3}\right) ; \mathrm{IR}\left(\mathrm{cm}^{-1}\right): 3068-2822(\mathrm{~m}, \mathrm{C}-$ H), 2222 (m, CN), 1732 (s, C=O), 1606 (C=C), 1247 (s, C-O-C), 942 (s, C-H out of 
plane). Anal. Calcd. for $\mathrm{C}_{12} \mathrm{H}_{10} \mathrm{ClNO}_{2}$ : C, 61.16; H, 4.28; N, 5.94; Found: C, 61.12; H, 4.25; N, 5.94.

\subsection{Ethyl 2-cyano-3-(3-chlorophenyl)-2-propenoate}

Yield 74\%; mp 108.2C ${ }^{\circ} \mathrm{C} ;{ }^{1} \mathrm{H}-\mathrm{NMR} \delta 8.2(\mathrm{~s}, 1 \mathrm{H}, \mathrm{CH}=), 8.0-7.0(\mathrm{~m}, 4 \mathrm{H}, \mathrm{Ph}), 4.3(\mathrm{t}, 2 \mathrm{H}$, $\left.\mathrm{OCH}_{2}\right), 1.3\left(\mathrm{t}, 3 \mathrm{H}, \mathrm{OCH}_{2} \underline{\mathrm{H}}_{3}\right) ;{ }^{13} \mathrm{C}-\mathrm{NMR} \delta 163(\mathrm{C}=\mathrm{O}), 154(\mathrm{HC}=), 135,133,130,128$ (Ph), $116(\mathrm{CN}), 103(\mathrm{C}=), 61\left(\mathrm{OCH}_{2}\right), 14\left(\mathrm{OCH}_{2} \underline{\mathrm{CH}}_{3}\right)$; IR $\left(\mathrm{cm}^{-1}\right): 3017-2819(\mathrm{~m}, \mathrm{C}-\mathrm{H})$, 2224 (m, CN), 1741 (s, C=O), 1568 (C=C), 1225 (s, C-O-C), 874 (s, C-H out of plane). Anal. Calcd. for $\mathrm{C}_{12} \mathrm{H}_{10} \mathrm{ClNO}_{2}$ : C, 61.16; H, 4.28; N, 5.94; Found: C, 60.89; H, 4.24; N, 5.80 .

\subsection{Ethyl 2-cyano-3-(4-chlorophenyl)-2-propenoate}

Yield 78\%; mp 99.9C $\mathrm{C}^{\circ}{ }^{1} \mathrm{H}-\mathrm{NMR} \delta 8.2(\mathrm{~s}, 1 \mathrm{H}, \mathrm{CH}=), 7.9-7.0(\mathrm{~m}, 4 \mathrm{H}, \mathrm{Ph}), 4.3(\mathrm{t}, 2 \mathrm{H}$, $\left.\mathrm{OCH}_{2}\right), 1.3\left(\mathrm{t}, 3 \mathrm{H}, \mathrm{OCH}_{2} \underline{\mathrm{C}}_{3}\right) ;{ }^{13} \mathrm{C}-\mathrm{NMR} \delta 163(\mathrm{C}=\mathrm{O}), 153(\mathrm{HC}=), 138,132,129(\mathrm{Ph})$, $116(\mathrm{CN}), 114(\mathrm{C}=), 61\left(\mathrm{OCH}_{2}\right), 14\left(\mathrm{OCH}_{2} \underline{\mathrm{CH}_{3}}\right) ; \mathrm{IR}\left(\mathrm{cm}^{-1}\right): 3020-2861(\mathrm{~m}, \mathrm{C}-\mathrm{H}), 2223$ (m, CN), 1741 (s, C=O), $1584(\mathrm{C}=\mathrm{C}), 1220$ (s, C-O-C), 811, 757 (s, C-H out of plane). Anal. Calcd. for $\mathrm{C}_{12} \mathrm{H}_{10} \mathrm{ClNO}_{2}$ : C, 61.16; H, 4.28; N, 5.94; Found: C, 61.09; H, 4.24; N, 5.90 .

\subsection{Ethyl 2-cyano-3-(2-fluorophenyl)-2-propenoate}

Yield 90\%; mp 55.7C $\mathrm{C}^{\circ}{ }^{1} \mathrm{H}-\mathrm{NMR} \delta 8.2(\mathrm{~s}, 1 \mathrm{H}, \mathrm{CH}=), 7.9-7.0(\mathrm{~m}, 4 \mathrm{H}, \mathrm{Ph}), 4.3(\mathrm{t}, 2 \mathrm{H}$, $\left.\mathrm{OCH}_{2}\right), 1.3\left(\mathrm{t}, 3 \mathrm{H}, \mathrm{OCH}_{2} \mathrm{CH}_{3}\right) ;{ }^{13} \mathrm{C}-\mathrm{NMR} \delta 163(\mathrm{C}=\mathrm{O}), 152(\mathrm{HC}=), 141,131,130,125$, $119(\mathrm{Ph}), 116(\mathrm{CN}), 105(\mathrm{C}=), 61\left(\mathrm{OCH}_{2}\right), 14\left(\mathrm{OCH}_{2} \underline{\mathrm{CH}_{3}}\right)$; IR $\left(\mathrm{cm}^{-1}\right): 3051-2841(\mathrm{~m}, \mathrm{C}-$ H), 2223 (m, CN), 1741 (s, C=O), 1592 (C=C), 1227 (s, C-O-C), 865 (s, C-H out of 
plane). Anal. Calcd. for $\mathrm{C}_{12} \mathrm{H}_{10} \mathrm{FNO}_{2}$ : C, 65.75; H, 4.60; N, 6.39; Found: C, 65.64; H, $4.22 ; \mathrm{N}, 6.36$.

\subsection{Ethyl 2-cyano-3-(3-fluorophenyl)-2-propenoate}

Yield 73\%; mp $103.2^{\circ} \mathrm{C} ;{ }^{1} \mathrm{H}-\mathrm{NMR} \delta 8.2(\mathrm{~s}, 1 \mathrm{H}, \mathrm{CH}=), 7.8-7.0(\mathrm{~m}, 4 \mathrm{H}, \mathrm{Ph}), 4.3(\mathrm{t}, 2 \mathrm{H}$, $\left.\mathrm{OCH}_{2}\right), 1.3\left(\mathrm{t}, 3 \mathrm{H}, \mathrm{OCH}_{2} \mathrm{CH}_{3}\right) ;{ }^{13} \mathrm{C}-\mathrm{NMR} \delta 164(\mathrm{C}=\mathrm{O}), 154(\mathrm{HC}=), 162,135,130,127$, $120(\mathrm{Ph}), 116(\mathrm{CN}), 103(\mathrm{C}=), 68\left(\mathrm{OCH}_{2}\right), 14\left(\mathrm{OCH}_{2} \underline{\mathrm{CH}_{3}}\right)$; IR $\left(\mathrm{cm}^{-1}\right): 3062-2842(\mathrm{~m}, \mathrm{C}-$ H), 2221 (m, CN), 1730 (s, C=O), $1580(\mathrm{C}=\mathrm{C}), 1262$ (s, C-O-C), 931, 853 (s, C-H out of plane). Anal. Calcd. for $\mathrm{C}_{12} \mathrm{H}_{10} \mathrm{FNO}_{2}$ : C, 65.75; H, 4.60; N, 6.39; Found: C, 65.61; H, $4.59 ; \mathrm{N}, 6.29$.

\subsection{Propyl 2-cyano-3-(4-fluorophenyl)-2-propenoate}

Yield $87 \%$; mp $105.3^{\circ} \mathrm{C} ;{ }^{1} \mathrm{H}-\mathrm{NMR} \delta 8.2(\mathrm{~s}, 1 \mathrm{H}, \mathrm{CH}=), 8.1-7.1(\mathrm{~m}, 4 \mathrm{H}, \mathrm{Ph}), 4.3(\mathrm{t}, 2 \mathrm{H}$, $\left.\mathrm{OCH}_{2}\right), 1.3\left(\mathrm{t}, 3 \mathrm{H}, \mathrm{OCH}_{2} \mathrm{CH}_{3}\right) ;{ }^{13} \mathrm{C}-\mathrm{NMR} \delta 163(\mathrm{C}=\mathrm{O}), 154(\mathrm{HC}=), 132,129,116(\mathrm{Ph})$, $116(\mathrm{CN}), 109(\mathrm{C}=), 61\left(\mathrm{OCH}_{2}\right), 10\left(\mathrm{OCH}_{2} \underline{\mathrm{CH}}_{3}\right) ; \mathrm{IR}\left(\mathrm{cm}^{-1}\right): 3042-2812(\mathrm{~m}, \mathrm{C}-\mathrm{H}), 2221$ (m, CN), 1728 (s, C=O), 1608 (C=C), 1214 (s, C-O-C), 810 (m, C-H out of plane). Anal. Calcd. for $\mathrm{C}_{12} \mathrm{H}_{10} \mathrm{FNO}_{2}$ : C, 65.75; H, 4.60; N, 6.39; Found: C, 65.88; H, 4.46; N, 6.34.

\subsection{Ethyl 2-cyano-3-(2,3-dichlorophenyl)-2-propenoate}

Yield 82\%; mp 82.0C ${ }^{\circ} \mathrm{C} ;{ }^{1} \mathrm{H}-\mathrm{NMR} \delta 8.1$ (s, $\left.1 \mathrm{H}, \mathrm{CH}=\right), 8.0-7.0(\mathrm{~m}, 3 \mathrm{H}, \mathrm{Ph}), 4.3(\mathrm{t}, 2 \mathrm{H}$, $\left.\mathrm{OCH}_{2}\right), 1.3\left(\mathrm{t}, 3 \mathrm{H}, \mathrm{OCH}_{2} \underline{\mathrm{CH}}_{3}\right) ;{ }^{13} \mathrm{C}-\mathrm{NMR} \delta 163(\mathrm{C}=\mathrm{O}), 153(\mathrm{HC}=), 134,133,130,128$ $(\mathrm{Ph}), 116(\mathrm{CN}), 108(\mathrm{C}=), 61\left(\mathrm{OCH}_{2}\right), 14\left(\mathrm{OCH}_{2} \underline{\mathrm{CH}}_{3}\right) ; \mathrm{IR}\left(\mathrm{cm}^{-1}\right): 3012-2811(\mathrm{~m}, \mathrm{C}-\mathrm{H})$, 2222 (m, CN), 1740 (s, C=O), 1562 (C=C), 1221 (s, C-O-C), 870 (s, C-H out of plane). 
Anal. Calcd. for $\mathrm{C}_{12} \mathrm{H}_{9} \mathrm{Cl}_{2} \mathrm{NO}_{2}$ : C, 53.36; H, 3.36; N, 5.19; Found: C, 53.33; H, 3.13; N, 5.09.

\subsection{Ethyl 2-cyano-3-(2,4-dichlorophenyl)-2-propenoate}

Yield 89\%; mp 84.73C $\mathrm{C}^{\circ}{ }^{1} \mathrm{H}-\mathrm{NMR} \delta 8.2(\mathrm{~s}, 1 \mathrm{H}, \mathrm{CH}=), 7.9-7.0(\mathrm{~m}, 3 \mathrm{H}, \mathrm{Ph}), 4.3(\mathrm{t}, 2 \mathrm{H}$, $\left.\mathrm{OCH}_{2}\right), 1.3\left(\mathrm{t}, 3 \mathrm{H}, \mathrm{OCH}_{2} \underline{\mathrm{H}}_{3}\right) ;{ }^{13} \mathrm{C}-\mathrm{NMR} \delta 163(\mathrm{C}=\mathrm{O}), 152(\mathrm{HC}=), 138,131,129(\mathrm{Ph})$, $116(\mathrm{CN}), 104(\mathrm{C}=), 61\left(\mathrm{OCH}_{2}\right), 14\left(\mathrm{OCH}_{2} \underline{\mathrm{CH}}_{3}\right) ; \mathrm{IR}\left(\mathrm{cm}^{-1}\right): 3020-2861(\mathrm{~m}, \mathrm{C}-\mathrm{H}), 2222$ (m, CN), 1740 (s, C=O), $1582(\mathrm{C}=\mathrm{C}), 1221$ (s, C-O-C), 810, 754 (s, C-H out of plane). Anal. Calcd. for $\mathrm{C}_{12} \mathrm{H}_{9} \mathrm{Cl}_{2} \mathrm{NO}_{2}$ : C, 53.36; H, 3.36; N, 5.19; Found: C, 53.42; H, 3.36; N, 5.07.

\subsection{Ethyl 2-cyano-3-(2,6-dichlorophenyl)-2-propenoate}

Yield 76\%; mp 73.7C $\mathrm{C}^{1} \mathrm{H}-\mathrm{NMR} \delta 8.1$ (s, 1H, CH=), 7.4, $7.2(\mathrm{~m}, 3 \mathrm{H}, \mathrm{Ph}), 4.3(\mathrm{t}, 2 \mathrm{H}$, $\left.\mathrm{OCH}_{2}\right), 1.3\left(\mathrm{t}, 3 \mathrm{H}, \mathrm{OCH}_{2} \underline{\mathrm{CH}}_{3}\right) ;{ }^{13} \mathrm{C}-\mathrm{NMR} \delta 163(\mathrm{C}=\mathrm{O}), 151(\mathrm{HC}=), 135,132,130,129$ (Ph), $116(\mathrm{CN}), 102(\mathrm{C}=), 61\left(\mathrm{OCH}_{2}\right), 14\left(\mathrm{OCH}_{2} \underline{\mathrm{CH}}_{3}\right) ; \mathrm{IR}\left(\mathrm{cm}^{-1}\right): 3018-2868(\mathrm{~m}, \mathrm{C}-\mathrm{H})$, 2224 (m, CN), 1742 (s, C=O), 1567 (C=C), 1227 (s, C-O-C), 813 (s, C-H out of plane). Anal. Calcd. for $\mathrm{C}_{12} \mathrm{H}_{9} \mathrm{Cl}_{2} \mathrm{NO}_{2}$ : C, 53.36; H, 3.36; N, 5.19; Found: C, 53.47; H, 3.32; N, 5.01 .

\subsection{Ethyl 2-cyano-3-(3,4-dichlorophenyl)-2-propenoate}

Yield 67\%; ${ }^{1} \mathrm{H}-\mathrm{NMR} \delta 8.2(\mathrm{~s}, 1 \mathrm{H}, \mathrm{CH}=), 8.0-7.4(\mathrm{~m}, 3 \mathrm{H}, \mathrm{Ph}), 4.3\left(\mathrm{t}, 2 \mathrm{H}, \mathrm{OCH}_{2}\right), 1.3(\mathrm{t}$, $\left.3 \mathrm{H}, \mathrm{OCH}_{2} \mathrm{CH}_{3}\right) ;{ }^{13} \mathrm{C}-\mathrm{NMR} \delta 163(\mathrm{C}=\mathrm{O}), 154(\mathrm{HC}=), 134,132,131,128(\mathrm{Ph}), 116(\mathrm{CN})$, $102(\mathrm{C}=), 61\left(\mathrm{OCH}_{2}\right), 14\left(\mathrm{OCH}_{2} \underline{\mathrm{CH}}_{3}\right) ; \mathrm{IR}\left(\mathrm{cm}^{-1}\right): 3024-2869$ (m, C-H), $2224(\mathrm{~m}, \mathrm{CN})$, 
$1742(\mathrm{~s}, \mathrm{C}=\mathrm{O}), 1576(\mathrm{C}=\mathrm{C}), 1212$ (s, C-O-C), 819 (s, C-H out of plane). Anal. Calcd. for $\mathrm{C}_{12} \mathrm{H}_{9} \mathrm{Cl}_{2} \mathrm{NO}_{2}$ : C, 53.36; H, 3.36; N, 5.19; Found: C, 53.27; H, 3.14; N, 5.12.

\subsection{Ethyl 2-cyano-3-(2,4-difluorophenyl)-2-propenoate}

Yield 79\%; mp 58.9C $\mathrm{C}^{\circ}{ }^{1} \mathrm{H}-\mathrm{NMR} \delta 8.3(\mathrm{~s}, 1 \mathrm{H}, \mathrm{CH}=), 8.1-7.0(\mathrm{~m}, 3 \mathrm{H}, \mathrm{Ph}), 4.3(\mathrm{t}, 2 \mathrm{H}$, $\left.\mathrm{OCH}_{2}\right), 1.3\left(\mathrm{t}, 3 \mathrm{H}, \mathrm{OCH}_{2} \underline{\mathrm{H}}_{3}\right) ;{ }^{13} \mathrm{C}-\mathrm{NMR} \delta 163(\mathrm{C}=\mathrm{O}), 152(\mathrm{HC}=), 135,131,130,119$, 112, $105(\mathrm{Ph}), 116(\mathrm{CN}), 102(\mathrm{C}=), 61\left(\mathrm{OCH}_{2}\right), 14\left(\mathrm{OCH}_{2} \underline{C H}_{3}\right) ; \mathrm{IR}\left(\mathrm{cm}^{-1}\right): 3028-2834$ (m, C-H), 2222 (m, CN), 1747 (s, C=O), 1543 (C=C), 1223 (s, C-O-C), 813 (s, C-H out of plane). Anal. Calcd. for $\mathrm{C}_{12} \mathrm{H}_{9} \mathrm{~F}_{2} \mathrm{NO}_{2}$ : C, 60.76; H, 3.82; N, 5.90; Found: C, 60.69; H, $3.61 ; \mathrm{N}, 5.87$.

\subsection{Ethyl 2-cyano-3-(2-bromo-3,4-dimethoxyphenyl)-2-propenoate}

Yield $87 \%$; ${ }^{1} \mathrm{H}-\mathrm{NMR} \delta 8.1(\mathrm{~s}, 1 \mathrm{H}, \mathrm{CH}=), 7.5,6.9,(\mathrm{~m}, 2 \mathrm{H}, \mathrm{Ph}), 4.3\left(\mathrm{t}, 2 \mathrm{H}, \mathrm{OCH}_{2}\right), 3.8(\mathrm{~s}$, $\left.6 \mathrm{H}, \mathrm{PhOCH}_{3}\right), 1.3\left(\mathrm{t}, 3 \mathrm{H}, \mathrm{OCH}_{2} \mathrm{C}_{3}\right) ;{ }^{13} \mathrm{C}-\mathrm{NMR} \delta 163(\mathrm{C}=\mathrm{O}), 152(\mathrm{HC}=), 151,128,127$, $113(\mathrm{Ph}), 116(\mathrm{CN}), 107(\mathrm{C}=), 61\left(\mathrm{OCH}_{2}\right), 55\left(\mathrm{PhOCH}_{3}\right), 14\left(\mathrm{OCH}_{2} \underline{\mathrm{CH}_{3}}\right) ; \mathrm{IR}\left(\mathrm{cm}^{-1}\right)$ : 3008-2854 (m, C-H), 2223 (m, CN), 1747 (s, C=O), 1569 (C=C), 1227 (s, C-O-C), 818 (s, C-H out of plane). Anal. Calcd. for $\mathrm{C}_{14} \mathrm{H}_{14} \mathrm{BrNO}_{4}$ : C, 49.43; H, 4.15; N, 4.12; Found: C, 49.21; H, 3.85; N, 3.93.

\section{Copolymerization}

An attempted radical homopolymerization of the HECP compounds did not produce polymeric products similarly to other ring-substituted HECP [19]. Homopolymerization of vinyl acetate yielded 28.7 of poly(vinyl acetate) under these conditions [21]. 
Copolymerization (Sch. 1) of VAC and the halogen ring-substituted HECP resulted in formation of copolymers (Table 1) with weight-average molecular masses 5.2 to $9.2 \mathrm{kD}$.

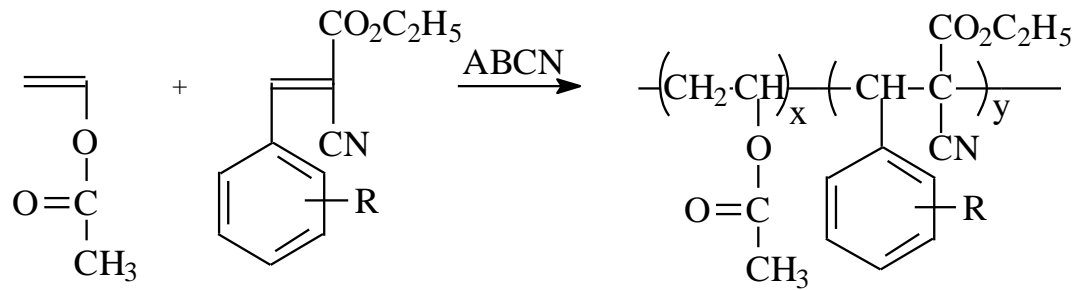

Scheme 1. Copolymerization of vinyl acetate and the ring-substituted ethyl 2-cyano-3phenyl-2-propenoates, $\mathrm{RPhCH}=\mathrm{C}(\mathrm{CN}) \mathrm{CO}_{2} \mathrm{C}_{2} \mathrm{H}_{5}$, where $\mathrm{R}$ is 2- $\mathrm{Br}, 3-\mathrm{Br}, 4-\mathrm{Br}, 2-\mathrm{Cl}, 3-\mathrm{Cl}$, 4-Cl, 2-F, 3-F, 4-F, 2,3-dichloro, 2,4-dichloro, 2,6-dichloro, 3,4-dichloro, 2,4-difluoro, 2bromo-3,4-dimethoxy.

According to the nitrogen elemental analysis, between 45.2 and $49.4 \mathrm{~mol} \%$ of TSE monomer is present in the copolymers prepared at VAC/HECP $=3(\mathrm{~mol})$, which is indicative of relatively high reactivity of the monomers towards VAC.

\section{Structure and Thermal Properties}

The structure of VAC-HECP copolymers was characterized by IR and NMR spectroscopy. A comparison of the spectra of the monomers, copolymers, and polyvinyl acetate with the spectra of ring-unsubstituted ethyl 2-cyano-3-phenyl-2-propenoate VAC [22] shows, that the reaction between the HECP monomers and VAC is a copolymerization. 
Table 1. Copolymerization of vinyl acetate and halogen ring-substituted ethyl 2-cyano-3phenyl-2-propenoates.

\begin{tabular}{|c|c|c|c|c|c|c|c|c|c|}
\hline & & & & & & \multicolumn{4}{|c|}{$T G A$} \\
\hline $\mathrm{R}$ & $\begin{array}{l}\text { Yield }^{\mathrm{a}} \\
\mathrm{wt} \%\end{array}$ & $\begin{array}{c}\mathrm{N} \\
\mathrm{wt} \%\end{array}$ & $\begin{array}{l}\text { HECP } \\
\text { in } \\
\text { pol., } \\
\text { mol\% }\end{array}$ & $\begin{array}{l}\mathrm{Mw} \\
\mathrm{kD}\end{array}$ & $\begin{array}{c}\mathrm{T}_{\mathrm{g}} \\
{ }^{\circ} \mathrm{C}\end{array}$ & $\begin{array}{l}\text { Onset of } \\
\text { decomp. } \\
{ }^{\circ} \mathrm{C}\end{array}$ & $\begin{array}{c}10 \% \\
\mathrm{wt} \\
\text { loss, } \\
{ }^{\circ} \mathrm{C}\end{array}$ & $\begin{array}{l}50 \% \\
\text { wt } \\
\text { loss, } \\
{ }^{\circ} \mathrm{C}\end{array}$ & $\begin{array}{c}\text { Residue } \\
\text { at } \\
500^{\circ} \mathrm{C}, \\
\text { wt } \%\end{array}$ \\
\hline 2-Bromo & 65.5 & 3.75 & 48.0 & 7.6 & 101 & 203 & 282 & 332 & 11.4 \\
\hline 3-Bromo & 56.8 & 3.68 & 46.1 & 6.2 & 105 & 211 & 276 & 312 & 10.2 \\
\hline 4-Bromo & 54.1 & 3.76 & 48.2 & 9.2 & 105 & 223 & 281 & 321 & 13.2 \\
\hline 2-Chloro & 52.1 & 4.14 & 45.7 & 5.9 & 115 & 219 & 286 & 338 & 15.2 \\
\hline 3-Chloro & 48.3 & 4.32 & 49.4 & 7.3 & 103 & 198 & 272 & 320 & 13.4 \\
\hline 4-Chloro & 53.2 & 4.17 & 46.3 & 9.1 & 102 & 201 & 271 & 334 & 11.5 \\
\hline 2-Fluoro & 67.2 & 4.44 & 47.2 & 7.4 & 95 & 209 & 271 & 339 & 13.1 \\
\hline 3-Fluoro & 71.1 & 4.51 & 48.5 & 8.8 & 98 & 205 & 274 & 332 & 12.2 \\
\hline 4-Fluoro & 34.4 & 4.34 & 45.4 & 6.4 & 114 & 208 & 258 & 331 & 12.3 \\
\hline 2,3-Dichloro & 56.2 & 3.67 & 43.6 & 7.5 & 107 & 206 & 265 & 326 & 14.7 \\
\hline 2,4-Dichloro & 67.2 & 3.76 & 45.7 & 5.2 & 101 & 159 & 227 & 331 & 13.0 \\
\hline 2,6-Dichloro & 56.2 & 3.81 & 46.9 & 5.7 & 115 & 187 & 268 & 301 & 14.3 \\
\hline 3,4-Dichloro & 45.7 & 3.74 & 45.2 & 7.2 & 112 & 191 & 269 & 321 & 10.8 \\
\hline 2,4-Difluoro & 67.2 & 4.12 & 45.5 & 8.2 & 110 & 227 & 278 & 335 & 8.8 \\
\hline $\begin{array}{l}\text { 2-Bromo-3,4- } \\
\text { dimethoxy }\end{array}$ & 56.3 & 3.21 & 47.2 & 7.2 & 112 & 201 & 276 & 323 & 11.4 \\
\hline
\end{tabular}




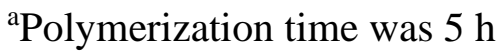

The copolymers prepared in the present work are all soluble in ethyl acetate, THF, DMF and $\mathrm{CHCl}_{3}$ and insoluble in methanol, ethyl ether, and petroleum ether. They are amorphous and show no crystalline DSC endotherm. Results of thermal analysis of VACHECP copolymers are presented in Table 1 . Relatively high Tg of the copolymers (95$115^{\circ} \mathrm{C}$ ) in comparison with that of polyvinyl acetate, $\mathrm{Tg}=28-31^{\circ} \mathrm{C}[23]$ indicates decrease of chain mobility of the copolymer due to the high dipolar character of the HECP structural units. Information on the degradation of the copolymers was obtained from thermogravimetric analysis. Decomposition of the copolymers in nitrogen occurred in two steps, apparently due to acetic acid elimination [24] in $160-340^{\circ} \mathrm{C}$ range followed by more slow decomposition of formed residue (15.1-1.5 wt\%), which then decomposed in the $500-650^{\circ} \mathrm{C}$ range.

\section{Conclusions}

Ring-substituted ethyl 2-cyano-3-phenyl-2-propenoates, $\mathrm{RPhCH}=\mathrm{C}(\mathrm{CN}) \mathrm{CO}_{2} \mathrm{C}_{2} \mathrm{H}_{5}$ ( $\mathrm{R}$ is 2$\mathrm{Br}, 3-\mathrm{Br}, 4-\mathrm{Br}, 2-\mathrm{Cl}, 3-\mathrm{Cl}$, 4-Cl, 2-F, 3-F, 4-F, 2,3-dichloro, 2,4-dichloro, 2,6-dichloro, 3,4dichloro, 2,4-difluoro, 2-bromo-3,4-dimethoxy) were prepared and copolymerized with vinyl acetate. The compositions of the copolymers were calculated from nitrogen analysis and the structures were analyzed by IR, $\mathrm{H}^{1}$ and ${ }^{13} \mathrm{C}-\mathrm{NMR}$. Thermal gravimetric analysis 
indicated that the copolymers decompose in in two steps, first in the $159-500^{\circ} \mathrm{C}$ range with residue (8.8-15.2 wt $\%)$, which then decomposed in the $500-650^{\circ} \mathrm{C}$ range.

\section{Acknowledgments}

The authors are grateful to acknowledge that the project was partly supported by the Coatings Industry Education Fund and Chicago Society of Coatings Technology.

\section{References}

[1] Straightforward preparation of highly loaded MWCNT-polyamine hybrids and their application in catalysis. Campisciano, Vincenzo; Burger, Rene; Calabrese, Carla; Liotta, Leonarda Francesca; Lo Meo, Paolo; Gruttadauria, Michelangelo; Giacalone, Francesco. Nanoscale Advances (2020), 2(9), 4199-4211.

[2] Synthesis of thiazacridine derivatives as anticancer agents against breast and hematopoietic neoplastic cells. Galdino-Pitta, Marina R.; Pereira, Michelly C.; Quirino, Michael W. L.; Rego, Moacyr J. B. M.; Lima, Maria do Carmo A.; Pitta, Maira G. R.; Pitta, Ivan R. Latin. American Journal of Pharmacy (2017), 36(1), 59-67.

[3] A Synthesis of Novel Selenophenes from Activated Acetylenes, Ethyl 2-Cyano-3arylacrylate and Potassium Selenocyanate. Siroos, Zahra; Hassanabadi, Alireza; Mosslemin, Mohammad H. Organic Preparations and Procedures International (2021), 53(2), 145-150. [4] Straightforward preparation of highly loaded MWCNT-polyamine hybrids and their application in catalysis. Campisciano, Vincenzo; Burger, Rene; Calabrese, Carla; Liotta, 
Leonarda Francesca; Lo Meo, Paolo; Gruttadauria, Michelangelo; Giacalone, Francesco.

Nanoscale Advances (2020), 2(9), 4199-4211.

[5] Design, Synthesis, Anti-Proliferative Evaluation and Cell Cycle Analysis of Hybrid 2-

Quinolones. Mohamed, Heba A. E.; Al-Shareef, Hossa F. Anti-Cancer Agents in Medicinal Chemistry (2019), 19(9), 1132-1140.

[6] Novel synthesis of 3-cyano-2-pyridones derivatives catalyzed by $\mathrm{Au}-\mathrm{Co} / \mathrm{TiO} 2$.

Mehiaoui, Nawel; Kibou, Zahira; Berrichi, Amina; Bachir, Redouane; Choukchou-Braham, Noureddine. Research on Chemical Intermediates (2020), 46(12), 5263-5280.

[7] Selenotungstates incorporating organophosphonate ligands and metal ions: synthesis, characterization, magnetism and catalytic efficiency in the Knoevenagel condensation reaction. Kong, Hui; He, Peipei; Yang, Zongfei; Xu, Qiaofei; Wang, Jiawei; Ban, Ran; Ma, Pengtao; Wang, Jingping; Niu, Jingyang. Dalton Transactions (2020), 49(22), 7420-7425.

[8] New Hybrid Organic-inorganic Multifunctional Materials Based on Polydopamine-like Chemistry. Calabrese, Carla; Liotta, Leonarda Francesca; Soumoy, Loraine; Aprile, Carmela; Giacalone, Francesco; Gruttadauria, Michelangelo. Asian Journal of Organic Chemistry (2021), 10(11), 2932-2943.

[9] Optimized Monofluoromethylsulfonium Reagents for Fluoromethylene-Transfer Chemistry. Sperga, Arturs; Melngaile, Renate; Kazia, Armands; Belyakov, Sergey; Veliks, Janis. Journal of Organic Chemistry (2021), 86(4), 3196-3212.

[10] Discovery, stereospecific characterization and peripheral modification of 1-(pyrrolidin1-ylmethyl)-2-[(6-chloro-3-oxo-indan)-formyl]-1,2,3,4-tetrahydroisoquinolines as novel selective $\kappa$ opioid receptor agonists. Gan, Zong-Jie; Wang, Yu-Hua; Xu, Yun-Gen; Guo, 
Ting; Wang, Jun; Song, Qiao; Xu, Xue-Jun; Hu, Shi-Yuan; Wang, Yu-Jun; Wang, De-

Chuan; et al. Organic \& Biomolecular Chemistry (2015), 13(20), 5656-5673.

[11] Aerobic oxidative cleavage of $\mathrm{C}=\mathrm{C}$ bond to carbonyl compound. Jia, Long-Fei; $\mathrm{Li}$, Huai-Zhu; Li, Zhi-Hao; Li, Rui-Jun; Yang, Guan-yu. Results in Chemistry (2021), 3, 100137.

[12] Synthesis of thiazacridine derivatives as anticancer agents against breast and hematopoietic. Galdino-Pitta, Marina R.; Pereira, Michelly C.; Quirino, Michael W. L.; Rego, Moacyr J. B. M.; Lima, Maria do Carmo A.; Pitta, Maira G. R.; Pitta, Ivan R. Latin American Journal of Pharmacy (2017), 36(1), 59-67.

[13] Synthesis of some New Dihydropyrimidine Derivatives by Cyclization of Polarized Unsaturated Systems. Ragab, Islam; Selim, Yasser A.; Abd El-Azim, Mohamed H. M. Journal of Heterocyclic Chemistry (2018), 55(12), 2727-2731.

[14] Synthesis of multifunctional polymer containing Ni-Pd NPs via thiol-ene reaction for one-pot cascade reactions. Javad Kalbasi, Roozbeh; Mesgarsaravi, Niloofar; Gharibi, Reza. Applied Organometallic Chemistry (2019), 33(4),

[15] A highly efficient protocol for the regio- and stereo-selective synthesis of spiro pyrrolidine and pyrrolizidine derivatives by multicomponent reaction. Dandia, Anshu; Jain, Anuj K.; Laxkar, Ashok K.; Bhati, Dharmendra S. Tetrahedron Letters (2013), 54(24), 3180-3184.

[16] Preparation of indolyl acrylates and related compounds alone and in compositions and methods for modulating hair growth. Lowry, William E.; Jung, Michael E.; Christofk, Heather R.; Liu, Xiaoguang. PCT Int. Appl. (2020), WO 2020142413 A1 20200709. 
[17] Novel Copolymers of Styrene. 3. Halogen Ring-Substituted Ethyl 2-Cyano-3-Phenyl-2-

Propenoates. Kharas, Gregory B.; Molina, Eric S.; Abma, Kathleen E.; Ali, Roshan A.;

Bairaktaris, Kiki D.; Biasiello, Fred P.; Cygan, Eva A.; Gibson, Jami; Haq, Syed; Hoag,

Dawn E.; et al. Journal of Macromolecular Science, Part A: Pure and Applied Chemistry (2013), 50(2), 139-143.

[18] SciFinder structure search accessed Dec 16, 2021.

[19] Novel functional copolymers of vinyl acetate: 1. Alkyl ring-substituted ethyl 2cyano-3-phenyl-2-propenoates. Benjamin Y. Killam, Rima T. Barkauskas, Daniel P. Dembiec, Rebecca S. Farrell, Maritza A. Gallego, Kelly A. Kaiser, Michelle L. Keeling, Kari Y. Kang, Gretchen R. Verdoorn, and Gregory B. Kharas. ChemRxiv. Cambridge Open Engage Version 1 Sep 06, 2021. https://doi.org/10.33774/chemrxiv-2021-ksld6

[20] Smith, M. B., March, J. (2001) Addition to Carbon-Hetero Multiple Bonds, In March's Advanced Organic Chemistry; J. Wiley \& Sons: New York, Ch.16, 1225.

[21] Novel Copolymers of Vinyl Acetate with Alkyl and Alkoxy Ring-Disubstituted 2Phenyl-1,1-dicyanoethylenes. G.B. Kharas, S.M. Russell, A. Alshaikh, M.L. Jonathas, A.P. Reddy, A.A. Rusinak, Y. Tsang, R.A.Wilczak, A. Virani, V. Havalad, and E. Hanawa. J. Macromol.Sci., A45 (6) 416-419 (2008).

[22] Characterization of copolymers of vinyl acetate with ethyl $\alpha$-cyanocinnamate. G.B. Kharas and D.H. Kohn. J. Polym. Sci. Polym. Chem. Ed., 23, 577-582 (1984). 
[23] Characterization and physical properties of low molecular weight poly(vinyl acetate) and poly(vinyl alcohol). Toshiaki Sato and Takuji Okaya. Polymer Journal, Vol. 24, No.9, pp 849-856 (1992).

[24] The thermal degradation of poly(vinyl acetate) and poly(ethylene-co-vinyl acetate), Part I: Experimental study of the degradation mechanism. B. Rimez, H. Rahier, G. Van Assche, T. Artoos, M. Biesemans, B. Van Mele. Polymer Degradation and Stability, 93 (2008) 800-810. 